educate and support referral sources (caregivers and schools) on how to identify girls with autism is recommended and would be informed by further research focus on the previous point. Active case ascertainment should be considered in future research and follow-up of girls who do not receive a diagnosis at initial assessment were additional learning points that came from the review.

\section{Quality of sleep and burnout among undergraduate medical students at the university of Nairobi, Kenya \\ Linda Nyamute ${ }^{1 \star}$, Muthoni Mathai ${ }^{2}$ and Anne Mbwayo ${ }^{3}$ \\ ${ }^{1}$ University of Nairobi; ${ }^{2}$ Consultant Psychiatrist and Senior lecturer, University of Nairobi and ${ }^{3}$ Clinical psychologist and lecturer, \\ University of Nairobi \\ ${ }^{*}$ Corresponding author.}

doi: 10.1192/bjo.2021.742

Aims. The main objective was to determine whether quality of sleep is associated with burnout among undergraduate medical students at the University of Nairobi.

The null hypothesis in our study population was; 'There is no significant association between poor sleep quality and burnout'. Background. In a pressure prevailing environment, medical students find themselves in a vicious cycle of cutting down on sleep in attempts to cope and adjust to increasing workloads. Students with poor sleep quality have been found to perform worse in their board exam and have strained social engagements. Ultimately, this chronic sleep deprivation may lead to burnout which may cause diminished sense of accomplishment and impaired professional conduct, that may be carried on to the career as a physician. High levels of burnout have been associated with suicides.

Method. The sample size obtained was 384 and participants were selected by a mixed sampling method. Data collection was through self-administered questionnaires. Scales used for this study were the Pittsburg Sleep Quality Index(PSQI) and the Oldenburg Burnout Inventory(OLBI).

Ethical considerations were adhered to and approval obtained from the Kenyatta National Hospital-University of Nairobi (KNH-UON) Ethics Board. Data entry and analysis was by SPSS v23. Data from 336 questionnaires were deemed fit for analysis.

Result. With a response rate of $87.5 \%$, the prevalence of poor sleep quality and burnout were $69.9 \%$ and $74.7 \%$ respectively. There was a significant positive association between poor sleep quality and female gender, clinical years of study, living with family, poorly perceived socio-economic state and poor subjective academic performance. In addition, being female, younger, preclinical years, living independently off-campus and poor subjective academic performance were significantly associated with higher levels of burnout.

Burnout had a significant correlation with poor sleep quality. Daytime functioning, a component of sleep quality had the highest correlation with components of burnout, disengagement and exhaustion. Overall, $57 \%$ of the respondents had both poor sleep quality \&burnout, while only $12 \%$ were good sleepers with no burnout. Furthermore, having poor sleep increased the risk of having burnout by 2.8 times. It is crucial that students adopt better sleeping habits to reduce the risk of burnout.

Conclusion. With the high prevalence of poor sleep quality and burnout, peer-support groups and peer-led mentorship programs are recommended within this population to help deal with expectations, challenges and difficulties encountered within the course of medical education, in addition to preparing for the early future careers.
What is the prevalence of self-harming and suicidal behaviour in under 18s with autism spectrum disorder, with or without an intellectual disability?

Rosalind Oliphant*, Eleanor Smith and Victoria Grahame

Cumbria, Northumberland, Tyne And Wear NHS Foundation Trust ${ }^{\star}$ Corresponding author.

doi: 10.1192/bjo.2021.743

Aims. The aims of this systematic review are to summarise data on the prevalence of suicidal behaviours and self-harm in under $18 \mathrm{~s}$ with Autism Spectrum Disorder (ASD) and consider the impact of Intellectual Disability (ID). It was hypothesised that the prevalence of these behaviours may be higher in under $18 \mathrm{~s}$ with ASD than in the general population.

Background. In the general population, rates of self-harm and suicide in under $18 \mathrm{~s}$ are of increasing concern. Whilst there is an emerging evidence base considering suicidality in autistic adults, less in known about the experience of under 18s. There has been very little research focused on how self-harm seen within the general population presents in the context of ASD and whether it continues to be a predictor of future suicidal behaviour. This may be partly due to self-harm being considered alongside Self-Injurious Behaviours (SIB), which have long been recognised as part of the clinical presentation of ASD and may have other functions (e.g. fulfilling sensory stimulation needs).

Method. A systematic literature search was conducted in line with PRISMA guidelines. For this review, all papers that included data on prevalence of self-harm and/or suicidal behaviours in under $18 \mathrm{~s}$ with ASD were included. Studies that only reported on the prevalence of the broader entity of SIB (characterised as stereotypic or habitual) were excluded.

Result. 338 papers were initially identified and 9 met eligibility criteria. There was considerable variation in how different aspects of self-harm and suicidal behaviours were addressed between groups and also between population samples, making it difficult to generalise the findings. The prevalence of self-harming and suicidal behaviours ranged from $7 \%$ to $73 \%$, indicating that this is a clinically significant problem for this patient group. The only study that considered the impact of co-existing ID did not identify significant differences between groups (ID vs no ID).

Conclusion. There was variation in the reported prevalence rates but results suggested that rates of both self-harm and suicidal behaviour may be elevated in under $18 \mathrm{~s}$ with ASD compared to the general population. This is in keeping with literature relating to autistic adults but in contrast to conclusions of a previous systematic review. This review highlights the need for further research to explore the experience of self-harm and suicidal behaviour in autistic children and young people.

\section{A survey into the influence of dopaminergic drug exposure on 'sense of presence' symptoms in patients with parkinson's disease

\author{
Emma Padfield*, Hannah Potticary and Tim Segal \\ Kings College London, South London and Maudsley NHS \\ Foundation Trust \\ ${ }^{\star}$ Corresponding author.
}

doi: 10.1192/bjo.2021.744

Aims. The first objective was to estimate prevalence of sense of presence (SoP) experiences in patients with Parkinson's Disease (PD), including whether onset was prior to or after commencing 
dopaminergic medication. The second objective was to explore the relationship between frequency of SoP experiences and dopaminergic drug, drug dosage and length of drug exposure. The experimental hypothesis was that SoP symptoms in PD would present more frequently in patients treated longer and with higher dopaminergic drug doses.

Background. PD is a debilitating neurodegenerative disorder. Psychiatric symptoms are common and associated with impaired quality of life and higher treatment costs. PD psychosis often starts with 'minor hallucinations', the most common being a false 'sense of presence' (SoP), the vivid sensation that someone else is nearby when nobody is there. SoP symptoms typically do not cause significant distress but may act as a prognostic marker for future severe psychosis and may prompt alteration of treatment or reduction in dopaminergic drug dosage. This study aimed to extend prior research by characterizing SoP further and investigating the link with dopaminergic medication.

Method. This was a retrospective, cross-sectional study. Twenty-one patients diagnosed with PD completed a questionnaire to identify presence of SoP symptoms, duration of symptoms, timing of onset related to dopaminergic treatment and the frequency of symptoms in relation to current levodopa equivalent dose (LED). Descriptive frequencies were compared using a two-tailed t-test. Multiple regression analysis was conducted to assess the relationship between frequency of SP experiences, levodopa equivalent dose and length of drug exposure.

Result. Sixteen of twenty-one patients reported experiencing SoP symptoms. Patients who had not experienced SoP symptoms had a significantly lower LED than those who had experienced these symptoms. There were no other significant differences between the groups. No statistical significance was shown on regression analysis; however our study was not adequately powered for the regression analysis as the number of participants was too low.

Conclusion. This study confirms that SoP symptoms are common among patients with PD and supports a correlation between the total daily equivalent dose of levodopa and SoP symptoms. It does not provide evidence for a temporal relationship between onset of SoP symptoms and duration of dopaminergic treatment. The study was insufficiently powered and a larger study is required to investigate further.

\section{A two-year mirror-image study of the effect of treatment with paliperidone and aripiprazole long-acting injections on need for inpatient care and home treatment intervention

\author{
Shay-Anne Pantall ${ }^{1 *}$, Joseph Pilsbury ${ }^{2}$, Le Gan ${ }^{2}$ \\ and Lisa Brownell ${ }^{1}$ \\ ${ }^{1}$ Birmingham and Solihull Mental Health NHS Foundation Trust and \\ ${ }^{2}$ University of Birmingham \\ ${ }^{*}$ Corresponding author.
}

doi: 10.1192/bjo.2021.745

Aims. To evaluate the effect of the use of aripiprazole and paliperidone long acting injections on healthcare resource use

Background. Long acting injections of second-generation antipsychotics such as paliperidone and aripiprazole have become more commonly prescribed over the past decade. They have much higher acquisition costs when compared to first generation depot antipsychotics. It is therefore essential to demonstrate their tolerability and cost-effectiveness.

Method. We undertook an observational, retrospective two-year mirror study for all patients who started treatment with paliperidone long acting injection between January and June $2016(\mathrm{n}=47)$ or aripiprazole long acting injection between April 2014 and July $2017(\mathrm{n}=93)$. Clinical notes were examined to determine the number of admissions, inpatient days, home treatment episodes and number of home treatment days, in the 12 months preceding and following the commencement of the long acting injection.

Result. 70\% remained on paliperidone and $62 \%$ remained on aripiprazole at the end of the one-year period.

There was a significant reduction in occupied bed days in those treated with paliperidone from 78.2 days in the year before this treatment was started to 25.4 days in the year after $(p=0.002)$. There was a significant reduction in occupied bed days in those treated with aripiprazole from 66.51 days to 32.7 days $(p=0.0006)$.

There was no significant reduction in days spent under the care of home treatment teams for individuals treated with either of these medicines.

Conclusion. Treatment with either paliperidone or aripiprazole long-acting injection was associated with a reduction in admissions and occupied bed days of a magnitude that delivered an overall cost-saving despite the high drug acquisition costs. It remains to be determined how these reductions compare with other second-generation long-acting injections and firstgeneration depot antipsychotics.

\section{Using qualitative methods, what factors do child \&} adolescent higher trainees and new consultants (within 1 year of appointment), report in choosing their higher training subspecialty?

\section{Mary Parker*, Jim Boylan and Nicholas Wolstenholme}

Tees, Esk \& Wear Valley NHS Foundation Trust

${ }^{\star}$ Corresponding author.

doi: 10.1192/bjo.2021.746

Aims. This Research aims to identify factors reported by recent and current trainees in choosing Child \& Adolescent (C\&A) Psychiatry for their higher training/career path. The hypothesis is that via thematic analysis prominent factors will emerge to inform future recruitment and retention.

Background. The need to attract more doctors into Psychiatry has been identified by NHS, Royal College of Psychiatrists and the Media; Health Education England figures show core psychiatry had lowest fill rates of any specialty in $2016,17 \& 18$.

Some subspecialties experience particular difficulties e.g. C\&A. Royal College of Psychiatry analysis of workforce in March 2018 showed the numbers of C\&A psychiatrists of all grades have fallen by $6.3 \%$ in four years and for consultants alone the decrease was $6.9 \%$ over the same period.

However, very little research has been completed investigating why trainees might choose C\&A Psychiatry with a literature search revealing only one report of C\&A trainees views in the UK in 2006.

Method. A qualitative design was chosen to provide insight into the factors affecting participants in choosing their career.

The theoretical framework supporting the study relates to capturing experience via a case study approach, aiming to explore reported issues in a real life context and considering similarities in the cases to inform future recruitment.

The Research was approved by The Health Research Authority and local Research \& Development departments.

Purposeful sampling was used with voluntary participation following informed consent and non-identifiable demographic data were collected and analysed quantatively.

Semi-structured interviews to saturation were conducted with fourteen $(\mathrm{N}=14)$ participants asked questions exploring their 